\title{
The Widom line of supercooled water
}

\author{
Giancarlo Franzese $^{1}$ and H Eugene Stanley ${ }^{2}$ \\ ${ }^{1}$ Departament de Física Fonamental, Universitat de Barcelona, Diagonal 647, \\ 08028 Barcelona, Spain \\ 2 Centre for Polymer Studies and Department of Physics, Boston University, \\ Boston, MA 02215, USA \\ E-mail: gfranzese@ub.edu
}

Received 16 January 2007

Published 25 April 2007

Online at stacks.iop.org/JPhysCM/19/205126

\begin{abstract}
Water can be supercooled to temperatures as low as $-92^{\circ} \mathrm{C}$, the experimental crystal homogeneous nucleation temperature $T_{\mathrm{H}}$ at $2 \mathrm{kbar}$. Within the supercooled liquid phase its response functions show an anomalous increase consistent with the presence of a liquid-liquid critical point located in a region inaccessible to experiments on bulk water. Recent experiments on the dynamics of confined water show that a possible way to understand the properties of water is to investigate the supercooled phase diagram in the vicinity of the Widom line (locus of maximum correlation length) that emanates from the hypothesized liquid-liquid critical point. Here we explore the Widom line for a Hamiltonian model of water using an analytic approach, and discuss the plausibility of the hypothesized liquid-liquid critical point, as well as its possible consequences, on the basis of the assumptions of the model. The present analysis allows us (i) to find an analytic expression for the spinodal line of the high-density liquid phase, with respect to the low-density liquid phase, showing that this line becomes flat in the $P-T$ phase diagram in the physical limit of a large number of available orientations for the hydrogen bonds, as recently seen in simulations and experiments (Xu et al 2005 Proc. Natl Acad. Sci. 102 16558); (ii) to find an estimate of the values for the hypothesized liquid-liquid critical point coordinates that compare very well with Monte Carlo results; and (iii) to show how the Widom line can be located by studying the derivative of the probability of forming hydrogen bonds with local tetrahedral orientation which can be calculated analytically within this approach.
\end{abstract}

(Some figures in this article are in colour only in the electronic version)

\section{Introduction}

Liquid water can be supercooled to far lower than its melting temperature. The experimental limit of stability of bulk liquid water, the crystal homogeneous nucleation temperature $T_{\mathrm{H}}$, is $-41^{\circ} \mathrm{C}$ at atmospheric pressure [1], and $-92^{\circ} \mathrm{C}$ at $2 \mathrm{kbar}$ [2]. 
If rapidly cooled below $T_{\mathrm{H}}$, water freezes into a glassy, amorphous solid [3], forming at low pressure a low-density amorphous (LDA) state [3] and at high pressure a highdensity amorphous (HDA) state [4], with a volume discontinuity between them of $\approx 27 \%$, comparable to that between crystalline ice I and ice VI [5-8]. By compressing HDA to over $0.95 \mathrm{GPa}$, consistent with computer simulations [9], water forms the very-high-density amorphous (VHDA) $[10,11]$ with a volume discontinuity of $11 \%$. At $-148{ }^{\circ} \mathrm{C}$ the three polyamorphs can be formed by compression in a stepwise process LDA-HDA-VHDA [12], but additional investigation is needed to clarify if these are real discontinuous transitions or just very sharp increases of densities. By heating amorphous ice, water crystallizes above a limiting temperature of spontaneous crystallization $T_{\mathrm{X}}$, about $-123^{\circ} \mathrm{C}$ at $P \simeq 0.1 \mathrm{GPa}$, and no liquid bulk water can be probed for $T_{\mathrm{X}}<T<T_{\mathrm{H}}$.

Confined water can be supercooled even in this range of temperatures, e.g., in plant fibres at $-47^{\circ} \mathrm{C}$ [13], which has inspired several experiments [14] on water under confinement (see e.g., [15-19]) and on hydration and surface water [20-22], with liquid water existing at temperatures as low as $-113{ }^{\circ} \mathrm{C}$ [18] at ambient pressure. At these extremely low temperatures interesting dynamical phenomena have been observed [19, 23, 24], suggesting the possible relation between water dynamics and the dynamics of biological macromolecules in water $[22,25]$.

It is under investigation if a comparison between the results for hydration water and the properties of bulk water could give insight into the mechanisms of the anomalous behaviour of water. One possible way of performing this research is to compare the experiments with simulations of water models. Many of these models are based on effective interaction potentials optimized to fit some water property [26]. However, the many parameters and interactions prevent from obtaining a simple picture of the physical mechanisms.

A complementary approach is to analyse schematic models, based on phenomenological considerations and with few parameters, to help in understanding the relevance of each parameter for the reproduced water properties [27]. Among these models those described by a Hamiltonian are particularly suitable for analytic calculations and Monte Carlo simulations. One of these models [28, 29], solved analytically and by simulations, suggests that the cooperativity of the hydrogen bond network is a key feature to understand the behaviour of supercooled water.

\subsection{The anomalous response functions of water}

In normal liquids, the response functions decrease with decreasing $T$. Examples are the isothermal compressibility $K_{T}$, the isobaric heat capacity $C_{P}$, or the thermal expansivity $\alpha_{P}$ (figure 1). This is consistent with the decrease with decreasing $T$ of the fluctuations of quantities such as volume $V$ or entropy $S$, that are proportional to these response functions.

At variance with normal liquids, for water the amplitudes of all these response functions increase by decreasing $T$ below a value which depends on the considered quantity. The increase of $K_{T}$ below $46^{\circ} \mathrm{C}$, of $C_{P}$ below $35^{\circ} \mathrm{C}$ and of $\left|\alpha_{P}\right|$ below $4{ }^{\circ} \mathrm{C}$, are consistent with power laws diverging to infinity at about $-45^{\circ} \mathrm{C}$, hinting at some sort of critical behaviour [30, 31, 34]. The negative value of $\alpha_{P}$ is related to the most famous anomaly of water: its density maximum at $4{ }^{\circ} \mathrm{C}$. Below this temperature the volume of water expands, causing phenomena as frozen water pipes breaking or iceberg floating.

\section{Structural analysis of supercooled water}

As recognized by Linus Pauling in 1939 [35], the distinguishing feature of water, compared to other chemically similar substances, is the preponderance of hydrogen bonds. Each water 

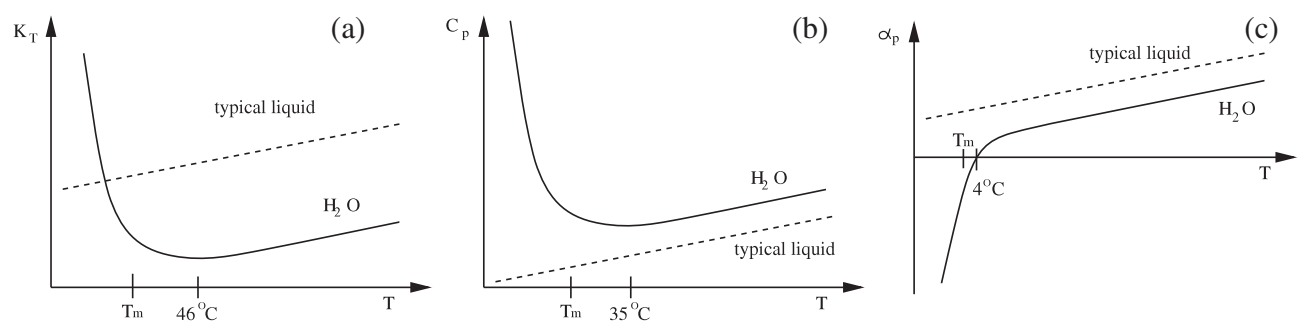

Figure 1. Dependence on temperature, at atmospheric pressure, of (a) the isothermal compressibility $K_{T} \equiv-(\partial \ln V / \partial P)_{T}$, proportional to the average value of the fluctuations of the volume per particle $\bar{V}$ through the relation $K_{T}=\left\langle(\delta \bar{V})^{2}\right\rangle / k_{\mathrm{B}} T \bar{V}$, with a minimum at $46{ }^{\circ} \mathrm{C}$ [30], (b) the constant-pressure specific heat $C_{P} \equiv T(\partial S / \partial T)_{P}$, proportional to the fluctuations of entropy $S$ through the relation $C_{P}=\left\langle(\delta S)^{2}\right\rangle / N k_{\mathrm{B}}$, where $N$ is the number of particles, with a minimum at $35^{\circ} \mathrm{C}$ [31], and (c) the thermal expansivity $\alpha_{P} \equiv(\partial \ln V / \partial T)_{P}$ proportional to the cross-fluctuations of entropy and volume, $\alpha_{P}=\langle(\delta S \delta \bar{V})\rangle / k_{\mathrm{B}} T \bar{V}$, with negative values below $4{ }^{\circ} \mathrm{C}$ [32]. The dashed line indicates the behaviour of a typical liquid, which, very roughly, is an extrapolation of the high- $T$ behaviour of liquid water. The anomalies become more striking below the melting temperature $T_{\mathrm{m}}$. Adapted from [33].

molecule has two hydrogen atoms and two lone electron pairs, forming on average four $\mathrm{O}-\mathrm{H}$ bonds with nearby $\mathrm{H}_{2} \mathrm{O}$ molecules. Experiments shows that each molecule is surrounded by four nearest neighbour molecules at a distance of about $2.75 \pm 0.05 \AA$, with a small dependence on $T$ and $P$ [36].

The first shell has a structure that resembles that of a tetrahedron. However, experiments [36] and simulations [37] show that the molecules bonded to the lone pairs of the central one can deviate from the tetrahedral position, having an uniform probability to be located at any intermediate angle, reflecting the delocalization of the lone pairs.

Neutron diffraction measurements, performed by Soper and Ricci in 2000 [36], show that by increasing $P$ from $26 \mathrm{MPa}$ (approximately 260 time the atmospheric pressure) to $400 \mathrm{MPa}$ at $T=268 \mathrm{~K}\left(-5.15^{\circ} \mathrm{C}\right)$ part of the second shell, originally tetrahedral and at an average distance of about $4.5 \AA$, collapses at a distance of about $3.5 \AA$ toward the first shell, increasing the local density. This structural change has been initially observed by varying $T$ from about 263 to $313 \mathrm{~K}$ in $\mathrm{x}$-ray structure factor experiments for heavy water $\mathrm{D}_{2} \mathrm{O}$ in 1983 [38]. Their data show that by decreasing $T$ the average $\mathrm{O}-\mathrm{O}-\mathrm{O}$ angle increases toward the tetrahedral angle $109.47^{\circ}$.

This structural change was already alluded as long ago as in 1951 by Pople's continuous random network model for water [39]. With his phenomenological model Pople shows that transforming water from ice to liquid there is a small, but non-negligible, probability of shift of second and third neighbour molecules toward the first coordination shell of a central molecule [39]. However, the possibility of a transformation with pressure and temperature from a low-density liquid (LDL) form of water to a high-density liquid (HDL) water has been seen for the first time in simulations for ST2 and TIP4P water by Poole et al in 1992 [40], showing the LDL-HDL phase transition below $200 \mathrm{~K}$ and above $150 \mathrm{MPa}$.

The continuous structural change is found also in ab initio water simulations [41] at very high pressure, $P=10^{4} \mathrm{MPa}$, and $T=600 \mathrm{~K}$. The result is qualitatively confirmed in simulations for TIP4P water [42] where the change occurs along a decreasing $P(T)$ line. In very good agreement with the experiment of Soper and Ricci, the change occurs at about 350 $400 \mathrm{MPa}$ at $T=268 \mathrm{~K}$ [42]. Further experimental confirmation has come in 2005 from sound velocity measurements [43] showing data consistent with the structural change at 290, 210 and $190 \mathrm{MPa}$ at 293, 316, $353 \mathrm{~K}$, respectively, in good agreement with the simulations [42]. 
The observed increase of density is reminiscent of the simulation results showing the presence of an interstitial molecules at a distance of 3.2-4.4 $\AA$ between the first and the second shell for SPC/E water [37] and for TIP3P water at the very high density of $0.1852 \mathrm{~mol}^{-1}$ [44], corresponding to a macroscopic density of $5.52 \mathrm{~g} \mathrm{~cm}^{-3}$, i.e., almost five times the estimated density $1.20 \mathrm{~g} \mathrm{~cm}^{-3}$ for HDL water [36]. These interstitial molecules are located in a region very close to the first shell and, in this sense, they increase the coordination number from 4 to 4.8 at $-10^{\circ} \mathrm{C}$, and to 5.0 at $100^{\circ} \mathrm{C}$ [37]. Therefore, the local density decreases on decreasing $T$.

The interstitial molecules could be partially $\mathrm{H}$ bonded to the central molecule [37], determining an overcoordination with bifurcated (shared) $\mathrm{H}$ bonds [45]. The overcoordination could be relevant to allow the reorientation of a molecule [46]. In liquid water the hydrogen bonds form a dynamic network, where hydrogen bonds have a lifetime of the order of picoseconds, with rotation and diffusion of the molecules [46]. The dynamics of the hydrogen bonds slows down when the temperature is decreased [47] before freezing in a full hydrogen bond network, corresponding to ice.

In many ices, e.g., ice Ih, Ic, VII, VIII, X, XI, for pressures below $200 \mathrm{MPa}$ and above $2000 \mathrm{MPa}$, the four nearest neighbours of the first coordination shell are at the tetrahedral angle $109.47^{\circ}$ [48]. This suggests that at very low $T$, just before freezing, the liquid assumes an approximately tetrahedral local structure.

This is, indeed, confirmed by experiments [36, 38] and ab initio simulations [41] where the open structure, at low $P$ and low $T$, is characterized by a $\mathrm{O}-\mathrm{O}-\mathrm{O}$ angle distribution peaked around the tetrahedral angle. The collapsed structure, at high $P$ and high $T$, instead has a distribution that is flatter or with more peaks. These results are consistent with previous experiments showing the gradual increase of the tetrahedral open structure at atmospheric pressure by decreasing $T$ from about 368 to $249 \mathrm{~K}$ for water [49] and from about 353 to $243 \mathrm{~K}$ in $\mathrm{H}_{2} \mathrm{O}-\mathrm{D}_{2} \mathrm{O}$ solution [50].

The following is a summary of the above observations.

(i) Below the homogeneous crystal nucleation line $T_{\mathrm{H}}$ some of the ice polymorphs possess a tetrahedral structure, or structures of copenetrating tetrahedra.

(ii) At low $P$ and low $T$, above $T_{\mathrm{H}}$, the molecules in supercooled water form an open approximately tetrahedral network of hydrogen bonds.

(iii) The open structure is characterized by a $\mathrm{O}-\mathrm{O}-\mathrm{O}$ angle distribution peaked around the tetrahedral angle $109.47^{\circ}$.

(iv) The open structure is prevalent at atmospheric pressure at $T$ as high as $400 \mathrm{~K}$ (about $\left.126^{\circ} \mathrm{C}\right)$.

(v) In the open structure the number of molecules in the first shell is on average four.

(vi) By increasing $P$, e.g., above about $300 \mathrm{MPa}$ at $300 \mathrm{~K}$, the number of molecules in the first coordination shell increases, with a partial collapse of the second (and maybe the third) neighbours toward the first neighbours, increasing the local density around the central molecule.

(vii) An analogous increase of local density, i.e. increase of number of molecules within the first and the second shell, occurs by increasing $T$, e.g., above $273 \mathrm{~K}$ at about $350 \mathrm{MPa}$.

(viii) The change between open and collapsed structure occurs at elevated pressures at low $T$ and at lower $P$ at higher $T$, i.e. occurs in an interval of $P$ that decreases for increasing $T$.

(ix) The local increase of density implies a distortion of the tetrahedral network, causing a broadening of the $\mathrm{O}-\mathrm{O}-\mathrm{O}$ angle distribution and the probable appearance of new peaks corresponding to interstitial molecules. 
(x) The interstitial molecules affect the strength of the tetrahedral hydrogen bonds, with the appearance of bifurcated bonds and overcoordination, favouring the breaking of the hydrogen bond and the diffusion of the molecules.

\section{A tractable model}

The above facts have inspired the formulation of a large variety of models [27, 51]. We consider here one proposed in [28, 29], studied in detail in [52] by a mean field analytic approach, and also by Monte Carlo simulations. In this section, we present a general formulation of the model, discussing in details its assumptions and their motivations. We will then present some specific results and perspectives.

The system is divided into cells $i \in[1, \ldots, N]$ on a regular square lattice, each containing a molecule, with a volume $v_{i} \geqslant v_{0}$, where $v_{0}$ is the hard-core volume of one molecule. In $d$ dimensions the distance between two nearest neighbour molecules $i$ and $j$ is $r_{i, j} \equiv$ $\left(v_{i}^{1 / d}+v_{j}^{1 / d}\right) / 2$. Since $v_{i}$ is a continuous variable, the distance $r_{i, j}$ is as well continuous.

The dimensionless density for the molecule in cell $i$ is $v_{0} / v_{i} \in(0,1]$. In the lattice gas spirit, we use a discrete two-state liquid index $n_{i}$ to quantify if the cell $i$ is in the liquid phase or not, with $n_{i}=1$ if $v_{0} / v \geqslant 0.5$ and $n_{i}=0$ otherwise.

The van der Waals attraction between the molecules is represented by a standard lattice gas Hamiltonian term

$$
\mathcal{H} \equiv-\epsilon \sum_{\langle i, j\rangle} n_{i} n_{j}
$$

where $\epsilon>0$ is the van der Waals attraction energy, which induces the liquid-gas phase transition.

Following [53], we assume that each water molecule can form four hydrogen bonds. As mentioned, see points $((\mathrm{v})-(\mathrm{x}))$ in previous section, this is no longer true at high $P$ and $T$ where a more dense, collapsed and distorted, local structure with bifurcated bonds is consistent with the data. However, we take into account the change of the local structure by (a) considering the possibility of breaking bonds or, better said, bifurcating and breaking bonds and (b) changing the local density as function of the number of non-bifurcated hydrogen bonds formed by a molecule, consistent with the lower density of the open structure, as in point (v) of previous section.

As suggested by point (x), bifurcated bonds decrease the strength of the network and favour the hydrogen bond breaking and re-formation. We simplify this situation by assuming that only non-bifurcated, i.e. normal, hydrogen bonds decrease the energy of the system. Hereafter we will say that a hydrogen bond is formed if it decreases the total energy and it is not bifurcated.

Two nearest neighbour molecules can form a hydrogen bond if the hydrogen of one is oriented toward the electron cloud of the other. Pictorially, it can be helpful to imagine that each molecule has four 'arms', two for the hydrogens and two for the electron clouds, and that oppositely charged arms of two nearest neighbour molecules must point to each other to form a hydrogen bond. However, this picture can be misleading, since, for example, the angle between the hydrogens (two 'arms') on the same molecule is not changing in a significant way with the formation of hydrogen bonds and only the electron clouds have some freedom to change their distribution around the oxygen.

Nevertheless, once a hydrogen bond is formed there is a reduction of the accessible configurations for the two molecules, because the molecules are less free to rotate. This reduction can be taken into account by adopting a variable with many states for each 'arm' and assuming that two facing arms have to be in the same state to form a hydrogen bond. At 
this level of description of the arms we do not distinguish between hydrogens and lone pairs. Their distinction would decrease further the number of accessible configurations.

To simplify the model we represent the arm of molecule $i$ facing molecule $j$ by the Potts variable $\sigma_{i j}=1, \ldots, q$, where $q$ is the maximum number of states for each arm and is a parameter of the model. Very large values of $q$, of the order of 100 , can be considered as an almost continuous variation of the arm position [53]. However, also lower values of $q$, of the order of 10, are enough to model the large reduction of accessible states for each arm forming a normal hydrogen bond.

Following [53], the energy decrease for the hydrogen bond formation is given by

$$
\mathcal{H}_{\mathrm{HB}} \equiv-J \sum_{\langle i, j\rangle} n_{i} n_{j} \delta_{\sigma_{i j}, \sigma_{j i}},
$$

where $J$ is the energy associated with each normal hydrogen bond formed $\left(\delta_{\sigma_{i j}, \sigma_{j i}}=1\right.$ if $\sigma_{i j}=\sigma_{j i}$ and $\delta_{\sigma_{i j}, \sigma_{j i}}=0$ otherwise). We assume that $0<J<\epsilon$ in such a way that hydrogen bonds are very rare in the gas phase.

To account for the change of local density when the structure goes toward the open structure by forming four hydrogen bonds tetrahedrally ordered, a small volume $v_{\mathrm{HB}}$ is added to the local volumes $v_{i}$ and $v_{j}$ of the two hydrogen bonded molecules $i$ and $j$, increasing their average distance to $r_{i j} \equiv\left(v_{i}+v_{j}+v_{\mathrm{HB}}\right) / 2$. Pictorially, this can be viewed as a local increase of the excluded volume associated with molecules $i$ and $j$ and is consistent with points ((ii), (v)) of previous section. If the system starts from low $T$ with an open network, in the $N P T$ ensemble the probability to keep a normal hydrogen bond between molecules $i$ and $j$, $\sim \exp \left[\left(J-P V_{i j}\right) / k_{\mathrm{B}} T\right]$, with $V_{i j} \equiv v_{i}+v_{j}$, decreases for increasing $T$ and for increasing $P$. Hence, the probability to have a larger excluded volume will decrease for increasing $T$ and $P$, inducing an increase of the local density, consistent with point ((vi), (vii)).

As a consequence of the local increase of volume for the formation of a normal hydrogen bond, the total volume $V \equiv \Sigma_{i} v_{i}$ increases and will be proportional to the total number of normal hydrogen bond $N_{\mathrm{HB}}$, as

$$
V \equiv V_{0}+N_{\mathrm{HB}} v_{\mathrm{HB}}
$$

where $V_{0}$ is the volume of the liquid with no hydrogen bonds, and

$$
N_{\mathrm{HB}} \equiv \sum_{\langle i, j\rangle} n_{i} n_{j} \delta_{\sigma_{i j}, \sigma_{j i}}
$$

We now use points ((i)-(iii), (ix)), specifically that the O-O-O angle formed by three nearest neighbour molecules becomes more locally tetrahedral when $T$ and $P$ decrease. We include this information in the model by introducing an intramolecular interaction between the arm variables on the same molecule. This intramolecular interaction induces a correlation between the arms such that they assume a locally tetrahedral structure at low $T$ and $P$. To this goal, it is sufficient to consider the Hamiltonian term

$$
\mathcal{H}_{\mathrm{IM}} \equiv-J_{\sigma} \sum_{i} n_{i} \sum_{(k, l)_{i}} \delta_{\sigma_{i k}, \sigma_{i l}}
$$

where $J_{\sigma}$ is the characteristic intramolecular energy interaction and the sum is extended over all the ${ }^{4} C_{2}=6$ different pairs $(k, l)_{i}$ of the arms of a molecule $i$. Here we assume that $0<J_{\sigma}<J$, in such a way that the intramolecular interaction is relevant only when hydrogen bonds are formed.

Therefore, our model has the total Hamiltonian

$$
\mathcal{H}_{\text {tot }} \equiv \mathcal{H}+\mathcal{H}_{\mathrm{HB}}+\mathcal{H}_{\mathrm{IM}}
$$

with the volume given by equation (3). 
The implicit form of the equation of state

$$
\mathcal{H}_{\mathrm{tot}}-T S+P V=\mu \sum_{i} n_{i}
$$

where $\mu$ is the chemical potential, by using equations (1)-(5) can be written as

$$
T S-P V_{0}=-\sum_{\langle i, j\rangle} \epsilon_{i j}^{\prime} n_{i} n_{j}-\sum_{i} \mu_{i}^{\prime} n_{i}
$$

where

$$
\mu_{i}^{\prime}(\sigma) \equiv \mu+J_{\sigma} \sum_{(k, l)_{i}} \delta_{\sigma_{k i}, \sigma_{l i}}, \quad J^{\prime} \equiv J-P v_{\mathrm{HB}} \quad \text { and } \quad \epsilon_{i j}^{\prime} \equiv \epsilon+J^{\prime} \delta_{\sigma_{i j}, \sigma_{j i}}
$$

are the effective chemical potential, effective hydrogen bond coupling and effective interaction energy, depending, respectively, on the arms configuration, on the pressure and on both pressure and arms configuration. From equation (9) is evident that by increasing $P$ the effective interaction decreases. Hence, we expect that by increasing $P$ at constant $T$, as well as by increasing $T$ at constant $P$, the probability of forming normal hydrogen bonds and their number $N_{\mathrm{HB}}$ decrease. Therefore, the density increases and the local tetrahedral order decreases, consistent with points ((vi)-(x)).

The molar Gibbs free energy is

$$
g \equiv u-T s+P v=\mu,
$$

where

$$
u \equiv \frac{\mathcal{H}_{\text {tot }}}{\sum_{i} n_{i}}, \quad v \equiv \frac{1}{\sum_{i} n_{i}} \quad \text { and } \quad s \equiv \frac{S_{W}+S_{\sigma}}{\sum_{i} n_{i}}
$$

are, respectively, the molar energy, the molar volume and the molar entropy, with the contribution $S_{W}$ of the $N$ variables $n_{i}$ and $S_{\sigma}$ of the $4 N$ variables $\sigma_{i j}$.

\subsection{Mean field analysis}

To find an analytic expression for $g$ we adopt a mean field analysis. The main assumption is that the system is in a homogeneous phase. As a consequence all the quantities are independent of the cell $i$. Hence, in our description we replace the cell volume $v_{i}$ with the average cell volume

$$
v \equiv \frac{\sum_{i} v_{i}}{N}
$$

the $r_{i j}$ distance with the average nearest neighbour distance $r=v^{1 / d}$ in $d$ dimensions and the cell liquid index $n_{i}$ with its average value

$$
n \equiv \frac{1}{N} \sum_{i} n_{i}
$$

In analogy with the average number density of liquid cells $n$, we introduce the average number density of arms in the appropriate state to form a normal hydrogen bond with local tetrahedral order

$$
n_{\sigma} \equiv \frac{1}{4 n N} \sum_{\langle i, j\rangle} \delta_{\sigma_{i j}, 1}
$$

where we assume that state $\sigma_{i j}=1$ corresponds to the arm in the locally tetrahedral configuration and the sum is performed only on the liquid cells. Hence, $n_{\sigma}=0$ for $n=0$.

Following the mean field random mixing approximation, we introduce a liquid-gas longrange order parameter $m \in[-1,1]$, defined as the difference between the number density of 
liquid cells and the number density of gas cells $m \equiv n-(1-n)$, and a locally tetrahedral hydrogen bond long-range order parameter $m_{\sigma} \in[0,1]$, defined as the difference between the number density of $\sigma_{i j}=1$ and the average number density for the other $q-1$ states $m_{\sigma} \equiv n_{\sigma}-\left(1-n_{\sigma}\right) /(q-1)$. Hence,

$$
n=\frac{1+m}{2}, \quad n_{\sigma}=\frac{1+(q-1) m_{\sigma}}{q},
$$

with $n=0$ for $m=-1$ while $n=1$ for $m=1$, and $n_{\sigma}=1 / q$ for $m_{\sigma}=0$ while $n_{\sigma}=1$ for $m_{\sigma}=1$.

Since every molecule has up to four nearest neighbours and four arms, we can write in the mean field approximation,

$$
\begin{aligned}
& \sum_{\langle i, j\rangle} n_{i} n_{j}=2 N n^{2}, \\
& \sum_{\langle i, j\rangle} n_{i} n_{j} \delta_{\sigma_{i j}, \sigma_{j i}}=2 N n^{2} p_{\sigma}, \\
& \sum_{i} n_{i} \sum_{(k, l)_{i}} \delta_{\sigma_{i k}, \sigma_{i l}}=6 N n p_{\sigma},
\end{aligned}
$$

where

$$
p_{\sigma} \equiv n_{\sigma}^{2}+(q-1)\left(\frac{1-n_{\sigma}}{q-1}\right)^{2}=\frac{1+(q-1) m_{\sigma}^{2}}{q}
$$

is the probability of having the facing arms of two nearest neighbour molecules in the same state, but not necessarily in the local tetrahedral order. Hence, the molar energy equation (11) becomes

$$
u=-2\left[\epsilon n+\left(J n+3 J_{\sigma}\right) p_{\sigma}\right]
$$

Defining $N_{+} \equiv n N, N_{-} \equiv N-N_{+}=N(1-n)$, and $N_{1} \equiv 4 n N n_{\sigma}, N_{i} \equiv$ $4 n N\left(1-n_{\sigma}\right) /(q-1)$ for $i=2, \ldots, q$, for the entropies $S_{W} \equiv k \ln \left[N ! /\left(N_{+} ! N_{-} !\right)\right]$and $S_{\sigma} \equiv k \ln \left[4 n N ! /\left(N_{1} ! N_{2} ! \ldots N_{q} !\right)\right]$, by using the Stirling approximation $\ln l !=l \ln l-l$, for any large integer $l$, we obtain

$$
\begin{aligned}
& -\frac{S_{W}}{k_{\mathrm{B}} N}=n \ln n+(1-n) \ln (1-n), \\
& -\frac{S_{\sigma}}{4 k_{\mathrm{B}} n N}=n_{\sigma} \ln n_{\sigma}+(q-1)\left(\frac{1-n_{\sigma}}{q-1}\right) \ln \left(\frac{1-n_{\sigma}}{q-1}\right) .
\end{aligned}
$$

Finally, using the mean field expression for the volume per cell equation (3), $V / N=$ $v_{0}+2 n v_{\mathrm{HB}} p_{\mathrm{HB}}$, we obtain the mean field molar liquid density $\rho \equiv n N / V$

$$
\rho=\frac{1+m}{2\left(v_{0} / n\right)+4 v_{\mathrm{HB}} p_{\mathrm{HB}}},
$$

where $v_{0} \equiv V_{0} / N$ and $p_{\mathrm{HB}} \equiv n^{2} p_{\sigma}$ is the probability of forming a normal hydrogen bond with local tetrahedral order between two nearest neighbour molecules.

The equations (15), (17), and (20) are consistent with the points ((vii), (ix), (x)) that suggest that there is no local tetrahedral order $\left(m_{\sigma} \rightarrow 0\right)$ for the high- $T$ liquid. Indeed, for the high- $T$ liquid is $p_{\sigma} \rightarrow 1 / q$ because there is no correlation between the states of the molecules arms, and hence $m_{\sigma} \rightarrow 0$ for equation (17). Therefore, if $m \geqslant 0$ (liquid phase), then $n \geqslant 1 / 2$, $p_{\mathrm{HB}} \geqslant 1 /(4 q)$ and $\rho v_{0} \leqslant 1 /\left[(2 / n)+v_{\mathrm{HB}} /\left(q v_{0}\right)\right]<1 / 2$.

On the other hand, points ((ii), (iii)) show that in the low- $T$ liquid phase $(m \rightarrow 1)$ the local tetrahedral order increases. This suggests two things. The first is that $m_{\sigma}$ increases toward 1 . 
When $m_{\sigma} \simeq 1 / 2, p_{\sigma} \simeq(q+3) /(4 q)<1, p_{\mathrm{HB}} \simeq p_{\sigma}$ and $\rho v_{0}>1 /\left[1+2 v_{\mathrm{HB}} / v_{0}\right]>1 / 2$. When $m_{\sigma} \rightarrow 1, p_{\sigma} \rightarrow 1, p_{\mathrm{HB}} \rightarrow 1$ and $\rho v_{0} \rightarrow 1 /\left[1+2 v_{\mathrm{HB}} / v_{0}\right]$. Hence, when the local tetrahedral order increases at low $T$, the density $\rho$ decreases, with a maximum at some intermediate $T$ at constant $P$, that is indeed the case of water.

The second observation suggested by points ((ii), (iii)), together with the finite value of the interaction constants $\epsilon, J$ and $J_{\sigma}$, is that locally tetrahedral configuration of one arm is favoured if already the other three arms of the same molecule are in the local tetrahedral state. This cooperative effect can be taken into account by introducing the mean field $h$ generated by these three arms and acting on the fourth arm of the same molecule. We choose $h$ proportional to the intramolecular interaction between the arms, the number of arms generating $h$ and to the density $n_{\sigma}$ of arms in the locally tetrahedral order, i.e.

$$
h \equiv 3 J_{\sigma} n_{\sigma} .
$$

We now approximate the quantity $p_{\sigma}$ calculated in the whole system with its value calculated for a single couple of facing interacting arms under the action of the mean field $h$, i.e.

$$
p_{\sigma}=\left\langle\delta_{\sigma_{i j}, \sigma_{j i}}\right\rangle_{h},
$$

where the right-hand side can be easily calculated as

$$
\left\langle\delta_{\sigma_{i j}, \sigma_{j i}}\right\rangle_{h}=\left[1+(q-1) \frac{2 w_{m_{\sigma}}+q-2}{\exp \left[\frac{J^{\prime}(P)}{k_{\mathrm{B}} T}\right]\left(w_{m_{\sigma}}^{2}+q-1\right)}\right]^{-1},
$$

with

$$
w_{m_{\sigma}} \equiv \exp \left\{\frac{3 J_{\sigma}\left[1+m_{\sigma}(q-1)\right]}{q k_{\mathrm{B}} T}\right\} .
$$

As expected for $p_{\sigma}$, also $\left\langle\delta_{\sigma_{i j}, \sigma_{j i}}\right\rangle_{h} \rightarrow 1 / q$ for $T \rightarrow \infty$ and $\left\langle\delta_{\sigma_{i j}, \sigma_{j i}}\right\rangle_{h} \rightarrow 1$ for $T \rightarrow 0$ and $J^{\prime}>0$, i.e. $P<J / v_{\mathrm{HB}}$ for equation (9).

For $P>J / v_{\mathrm{HB}}$ and $T \rightarrow 0,\left\langle\delta_{\sigma_{i j}, \sigma_{j i}}\right\rangle_{h} \rightarrow 0$. This is in apparent contradiction with $p_{\sigma}$ definition equation (17). In this case the effective hydrogen bond interaction is $J^{\prime}<0$, disfavoring the local tetrahedral ordering at low $T$. Hence $m_{\sigma} \rightarrow 0$ and, for equation (17), $p_{\sigma} \rightarrow 1 / q$. However, in the physical limit $q \rightarrow \infty$, we recover $p_{\sigma} \rightarrow 0$ as for $\left\langle\delta_{\sigma_{i j}, \sigma_{j i}}\right\rangle_{h}$. Therefore, the approximation in equation (22) includes all the physical conditions suggested by the previous observations ((i)-(x)) and holds better in the physical limit of large $q$.

We now can follow two strategies to minimize the Gibbs free energy $g$ :

(i) The first strategy, followed in [52], consists in replacing $p_{\sigma}$ for its approximation on the right-hand side of equation (22) in the mean field expression of $g$. Then, we numerically minimize $g$ with respect to $m$ and $m_{\sigma}$.

(ii) The second strategy, followed in [54], consists in solving, numerically, equation (22) with respect to $m_{\sigma}$ and calculating $p_{\sigma}$ from equation (17). Then, we numerically minimize $g$ with respect to the only free order parameter $m$. Since for $P>J / v_{\mathrm{HB}}$ the only possible solution is $m_{\sigma}=0$, and hence $p_{\sigma}=1 / q$, in this case we recover the results of the previous method only in the limit $q \rightarrow \infty$ [54]. However, even for finite $q$ the two methods for $P<J / v_{\mathrm{HB}}$ give the same qualitative phase diagram. Here we report new results with method (i).

First, the mean field results (figure 2(a)), consistent with computer simulations [52], reproduce the known phase diagram of fluid water, with the liquid-gas coexistence region ending in the critical point $C$, at $T_{C}=1.03 \pm 0.03$ and $P_{C}=0.18 \pm 0.04$, and with the temperatures of maximum density (TMD) at constant $P$ that decreases with increasing $P$ as in the experiments $[55,56]$. 

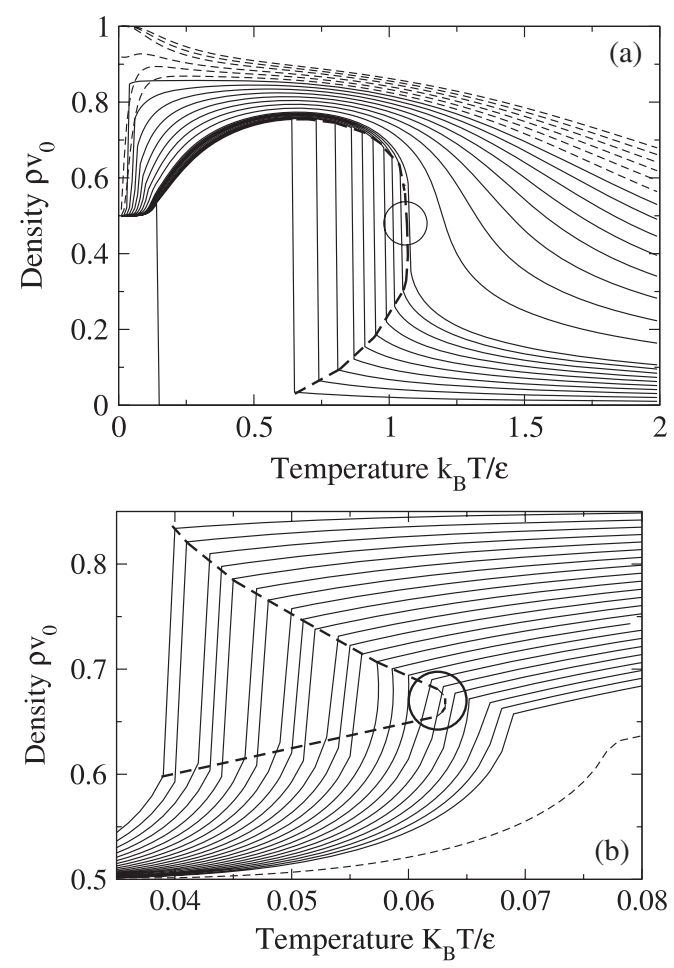

Figure 2. (a) Liquid density $\rho$ at constant pressure $P$ as function of temperature $T$. Continuous lines, from bottom to top, are for $0 \leqslant P v_{0} / \epsilon \leqslant 0.20$, with increment of 0.02 , and for $0.3 \leqslant P v_{0} / \epsilon \leqslant 1.0$, with increment of 0.1 . Thin dashed lines, from bottom to top, are for $1.1 \leqslant P v_{0} / \epsilon \leqslant 1.6$, with increment of 0.1 . The circle is the estimate of the liquid-gas critical point $C$, based on the discontinuity of the $T$-derivative of isobaric densities. For any $P \leqslant 1.3$ there is a temperature of maximum density (TMD) below which $\rho$ decreases, saturating at $0.5 v_{0}^{-1}$ at low- $P, T$. (b) Enlarged view at low $T$. Thin dashed line is for $P v_{0} / \epsilon=0.7$ and continuous lines, from bottom to top, are for $0.77 \leqslant P v_{0} / \epsilon \leqslant 0.99$, with increment of 0.01 . A discontinuity in $\rho$ appears above $P v_{0} / \epsilon=0.8$. The circle is the estimate of the hypothesized liquid-liquid critical point $C^{\prime}$, based on the discontinuity of the $T$-derivative of isobaric densities. In both panels the size of circle for the critical point is of the order of the error on the estimate and the thick dashed line is a guide for the eyes to mark the coexistence region with the discontinuity in $\rho$.

In the deeply supercooled region we find that the density has another discontinuity marking the coexistence region between two liquids at different densities. On decreasing $P$ the region terminates in the hypothesized liquid-liquid critical point $C^{\prime}$, at $k_{\mathrm{B}} T_{C^{\prime}} / \epsilon=0.062 \pm 0.02$ and $P_{C^{\prime}} v_{0} / \epsilon=0.82 \pm 0.02,{ }^{3}$ and following a line with negative slope in the $P-T$ phase diagram (figure 3 (b)), consistent with the liquid-liquid critical point scenario.

This picture was initially proposed by Poole et al on the basis of computer simulations [40] and then shown to be consistent with experiments with a discontinuous LDA-HDA transition [8]. In this scenario the LDA-HDA transition line does not terminate when it reaches the spontaneous crystallization line at $T_{\mathrm{X}}$, but extends above it, with LDA transforming without discontinuity into LDL, and HDA into HDL, giving a LDL-HDL phase transition line. According to this hypothesis, along this line the two kinds of liquids coexist as two separate phases. Since the collapsed structure of HDL is more disordered than the open structure of LDL, the entropy of HDL is larger than that of LDL and this implies, for thermodynamics

3 This estimate of $C^{\prime}$ is more consistent with the Monte Carlo result of [52] than the mean field estimate given in the same reference [52], based on calculations with lower precision than that presented here. 

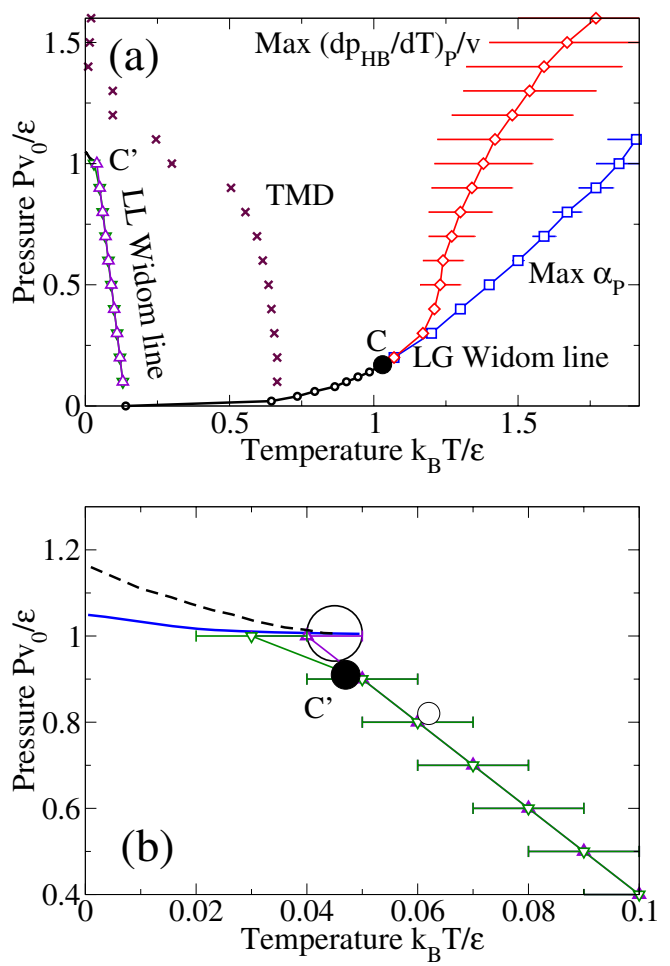

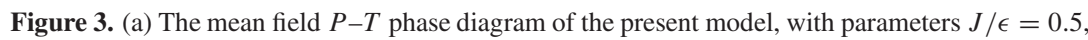

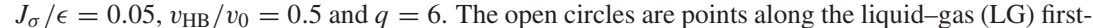

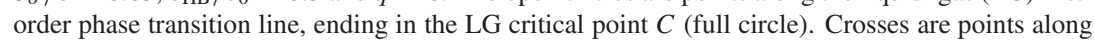

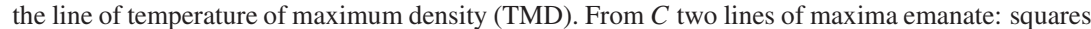

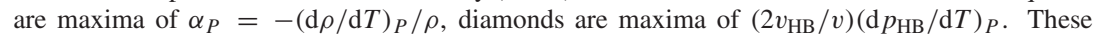

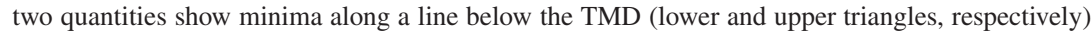

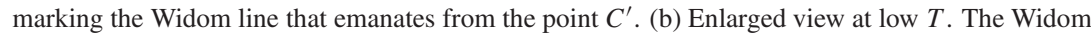

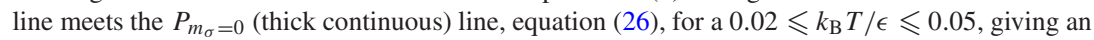

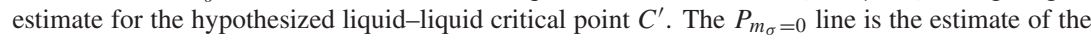

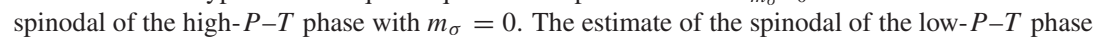

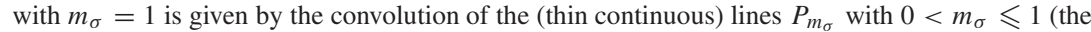

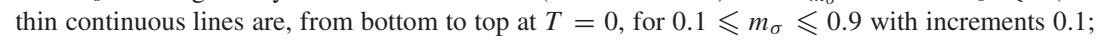

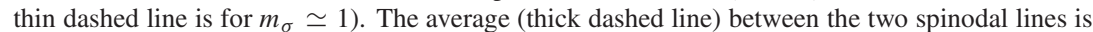

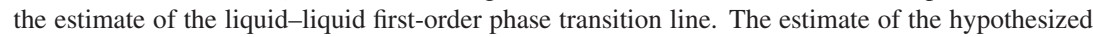

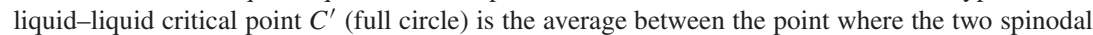

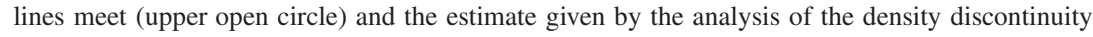

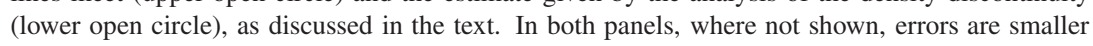

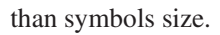

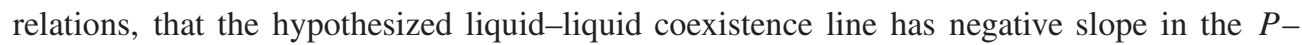

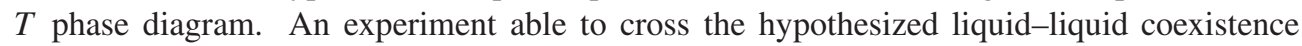

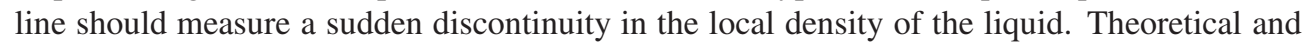

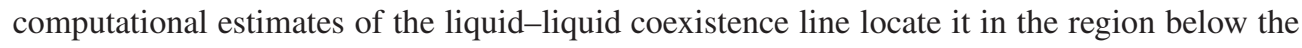

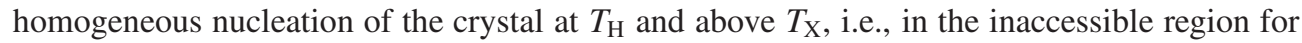

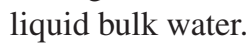

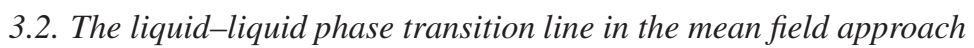

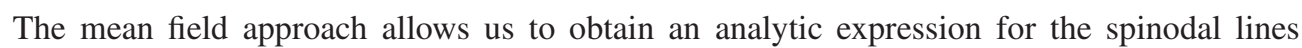

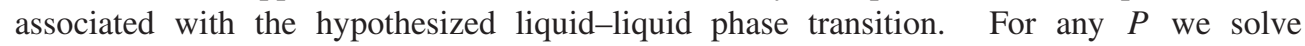


equation (22) with respect to $n_{\sigma}\left(m_{\sigma}, T, P\right)$. Then, to obtain the limit of stability $P_{m_{\sigma}=0}(T)$ of the high- $P-T$ HDL with no locally tetrahedral order $\left(m_{\sigma}=0\right)$ with respect to the low- $P-T$ LDL with locally tetrahedral order $\left(m_{\sigma}>0\right)$ we impose the condition

$$
n_{\sigma}\left(0, T, P_{m_{\sigma}=0}\right)=1 / q \text {. }
$$

The solution of equation (25) is

$$
P_{m_{\sigma}=0} v_{\mathrm{HB}}=J+k_{\mathrm{B}} T \ln \left[\frac{\left(\mathrm{e}^{3 J_{\sigma} /\left(q k_{\mathrm{B}} T\right)}\right)^{2}+(q-1)}{2 \mathrm{e}^{3 J_{\sigma} /\left(q k_{\mathrm{B}} T\right)}+(q-2)}\right],
$$

where $3 J_{\sigma} / q$ is the value of $h$, equation (21), for $m_{\sigma}=0$. In the limit $q \rightarrow \infty$ we recover $P_{m_{\sigma}=0}=J / v_{\mathrm{HB}}$ and the spinodal line becomes flat in the $P-T$ phase diagram. This behaviour is consistent with recent experimental results on confined water and associated simulations [57]. In [57] the behaviour of the HDL spinodal is related to the disappearing of a dynamical crossover in the experiments.

By imposing the condition

$$
n_{\sigma}\left(m_{\sigma}, T, P_{0}\right)=\frac{1+(q-1) m_{\sigma}}{q},
$$

we find $P_{m_{\sigma}}(T)$ for any $m_{\sigma}>0$ :

$$
P_{m_{\sigma}} v_{\mathrm{HB}}=J+k_{\mathrm{B}} T \ln \left[\frac{1-m_{\sigma}^{2}}{1+(q-1) m_{\sigma}^{2}} \frac{\left(\mathrm{e}^{3 J_{\sigma} /\left(q k_{\mathrm{B}} T\right)}\right)^{2+2(q-1) m_{\sigma}}+(q-1)}{2\left(\mathrm{e}^{3 J_{\sigma} /\left(q k_{\mathrm{B}} T\right)}\right)^{2+2(q-1) m_{\sigma}}+(q-2)}\right]
$$

that coincides with equation (26) for $m_{\sigma}=0$. In figure 3(b) we plot the lines $P_{m_{\sigma}}(T)$ for $m_{\sigma} \geqslant 0$. Their convolution give an estimate of the spinodal region and the vertex of this region at $k_{\mathrm{B}} T^{\prime} / \epsilon=0.045 \pm 0.005$ and $P^{\prime} v_{0} / \epsilon=1.006 \pm 0.001$ is an estimate of the liquidliquid critical point $C^{\prime}$. The latter estimate does not coincide with the one given in the previous section. To clarify this point and improve our understanding of the phase diagram we consider the Widom line, about which many paper have recently appeared [57-59] even though there is a long history of interest in this line [60].

\subsection{The Widom line and the estimate of the critical points}

The existence of a critical point induces large fluctuations in a region that extends to temperatures and pressures far away in the phase diagram. For example, experiments show that the effect of the gas-liquid critical point $C$ on the response functions is evident even at temperatures twice higher than the critical one. A similar behaviour is expected also for the hypothesized liquid-liquid critical point $C^{\prime}$.

Above the temperature of a critical point the thermodynamic response functions have an extreme (maximum or minimum) at the Widom line, defined as the locus of maximum correlation length in the $P-T$ phase diagram [61]. By decreasing $T$, the Widom line converges to the critical point, where the correlation length diverges together with the response functions. Therefore, for decreasing Talong the Widom line, the response functions show extremes that finally diverge at the critical point.

Since far above a critical point the maxima of correlation length and the extremes of response functions become smooth and flat, the Widom line is broadened in a region whose size increases at higher $T$. As a consequence, different response functions show extremes along different lines, all around the Widom line and all converging at the critical point or at higher $T$. The $T$ where they converge gives an upper limit of the critical temperature.

In figure 3 we show our mean field results for the loci of response functions above $C$ and above $C^{\prime}$. As response function we use here $\alpha_{P}$ and the $T$-derivative of the probability $p_{\mathrm{HB}}$ 

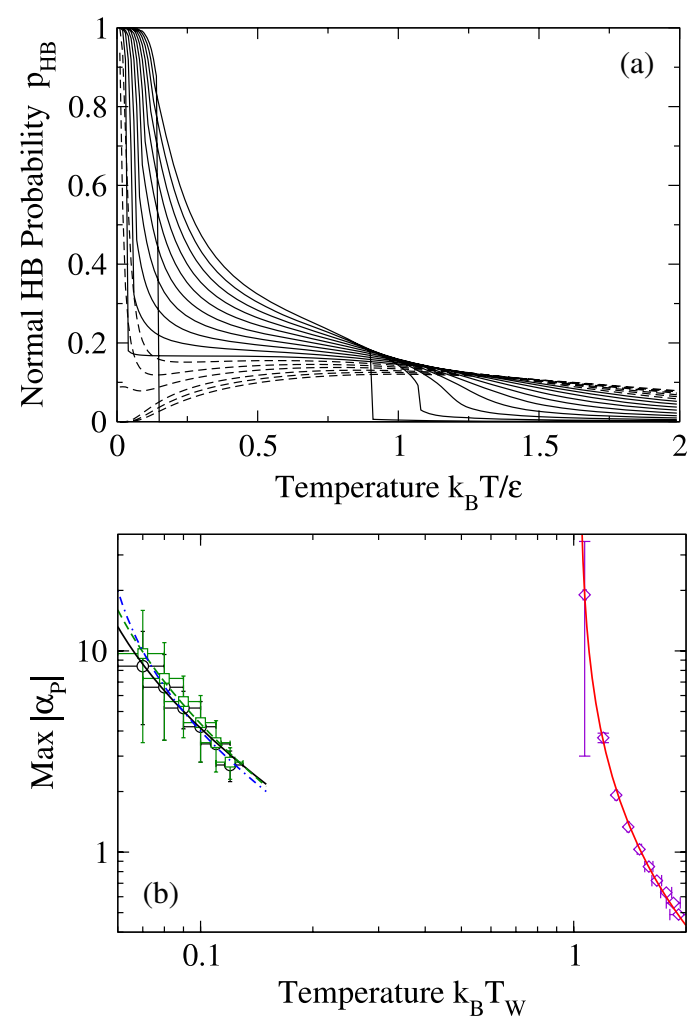

Figure 4. (a) The probability of forming a normal hydrogen bond with local tetrahedral order as a function of temperature $T$ at constant pressure $P$. From top to bottom at temperature 0.5, continuous lines are for $0 \leqslant P v_{0} / \epsilon \leqslant 1$ and dashed lines are for $1.1 \leqslant P v_{0} / \epsilon \leqslant 1.6$, with increment of 0.1 . The probability goes rapidly to 0 at low $P$ and high $T$, and has a maximum at $P$ above $P_{C^{\prime}}$, decreasing at low $T$. Below $P_{C^{\prime}}$, the probability $p_{\mathrm{HB}}$ increases toward 1 at low $T$, with a dramatic increase just below $P_{C^{\prime}}$. (b) Scaling of minima of $\alpha_{P}=-(1 / \rho)(\mathrm{d} \rho / \mathrm{d} T)_{P}$ (circles) and of $T$-derivative of $p_{\mathrm{HB}}$ at constant $P$ equation (29) (squares) for $T_{C^{\prime}}<T<T_{C}$ and of maxima of $\alpha_{P}$ (diamonds) for $T>T_{C}$. Lines are fits with power laws diverging at $k_{\mathrm{B}} T_{C} / \epsilon=1.026 \pm 0.005$ and $k_{\mathrm{B}} T_{C^{\prime}} / \epsilon=0.05 \pm 0.1$, with exponents $-1.22 \pm 0.02$ (continuous line for diamonds), $-0.7 \pm 0.3$ (continuous line for circles), $-0.9 \pm 0.3$ (dashed line for squares), $-1.0 \pm 0.1$ (dot-dashed line).

of forming a normal hydrogen bond with local tetrahedral order (figure 4(a)). The second is related to the first by the relation

$$
\alpha_{P}=2 \frac{v_{\mathrm{HB}}}{v}\left(\frac{\mathrm{d} p_{\mathrm{HB}}}{\mathrm{d} T}\right)_{P}+\frac{1}{v}\left(\frac{\mathrm{d}}{\mathrm{d} T} \frac{v_{0}}{n}\right)_{P}
$$

and we find, numerically, that the maxima of $\alpha_{P}$ coincide with those of the first term of equation (29) for $T_{C^{\prime}}<T<T_{C}$ and just above $T_{C}$. The deviation from $T \gg T_{C}$ is a consequence of the contribution to the thermal expansivity of the second term of equation (29), accounting for the fluctuations not related to the hydrogen bonding. The point where the different lines of maxima converge above $C$ are consistent with our estimate of $C$ (figure 3 ).

Knowing the location of the Widom line and the extremes of $\alpha_{P}$ and $\left(\mathrm{d} p_{\mathrm{HB}} / \mathrm{d} T\right)_{P}$, we can verify if the data are consistent with a power-law divergence at the estimated $T_{C}$ and $T_{C^{\prime}}$. As shown in figure 4 , the data for $T>T_{C}$ are well fitted by power laws with $k_{\mathrm{B}} T_{C} / \epsilon=1.026 \pm 0.005$ fully consistent with the estimate of the previous section. The large error bars on the maxima for $T_{C^{\prime}}<T<T_{C}$ do not allow us to extract easily an estimate of $T_{C^{\prime}}$, however the data are well consistent with fits where $T_{C^{\prime}}$ ranges from 0.04 to 0.06 , consistent 
with the estimates of the previous section. Hence, the analysis of the Widom line is consistent with a $k_{\mathrm{B}} T_{C^{\prime}} / \epsilon=0.05 \pm 0.01$.

By averaging all our present estimates for $C^{\prime}$, we conclude that $k_{\mathrm{B}} T_{C^{\prime}} / \epsilon=0.047 \pm 0.004$ and $P_{C^{\prime}} v_{0} / \epsilon=0.91 \pm 0.09$. These values compare very well with those $\left(k_{\mathrm{B}} T_{C^{\prime}} / \epsilon=\right.$ $0.045 \pm 0.005$ and $P_{C^{\prime}} v_{0} / \epsilon=0.841 \pm 0.042$ ) of our Monte Carlo simulations for the version of this model with a truncated Lennard-Jones potential for the van der Waals interaction [52]. A detailed numerical analysis of the Widom line and its relation with a change in the dynamics, based on Monte Carlo simulations is presented elsewhere [62].

\section{Discussion}

The results presented here, based on a Hamiltonian model for water, support the liquid-liquid phase transition hypothesis to interpret the anomalies of water. The anomalous increase of the response functions on cooling arises from approaching the liquid-liquid coexistence line, with a genuine divergence at the critical point.

This hypothesis is consistent with experiments [8] on the melting line of metastable ice IV and stable ice $\mathrm{V}$, showing an abrupt change in their slopes as predicted if it would intersect the metastable liquid-liquid phase transition line. However, the experimental resolution does not allow us to conclude whether the sharp change is a real discontinuity, as required by the liquid-liquid phase transition hypothesis. Interpolation of the experimental data is consistent with an estimation of the liquid-liquid critical point at $1 \mathrm{kbar}$ and $220 \mathrm{~K}$.

Nevertheless, the liquid-liquid phase transition hypothesis is not the only possible thermodynamically consistent interpretation. In the singularity-free interpretation [63, 53] LDL and HDL are still smoothly connected to LDA and HDA, respectively, but no phase transition separates them and a pressure increase give rise to a sharp, but continuous, increase of density. Hence, the large increase of response functions seen in the experiments represents only an apparent singularity, due to local density fluctuations, with no real divergence and all the anomalies are interpreted as a consequence of the negative volume-entropy crossfluctuations [53].

Our model coincides with the one proposed to support the singularity-free scenario [53] when we choose the intramolecular interaction $J_{\sigma}=0$. Preliminary results [54] show that for $J_{\sigma} \rightarrow 0$ the liquid-liquid critical point moves in a continuous way to $T=0$, leaving the rest of the phase diagram unaffected. Hence, our model suggests that the singularity-free scenario is valid only for zero intramolecular interaction, a condition that is not consistent with the experimental observations ((i)-(x)) and with first-principles simulations for water [64].

An indirect confirmation of this conclusion comes from studies of other locally tetrahedrally coordinated liquids that, like water [73], at low enough $T$ and $P$, have anticorrelated entropy and volume fluctuations, such as $\mathrm{SiO}_{2}$ and $\mathrm{GeO}_{2}$. Recent simulations are consistent with the intriguing possibility that silica and silicon may also display a liquidliquid critical point $[65,66]$.

Interestingly, some properties of water, such as the polymorphism or the existence of a low-density open crystal, could be present also in substances without a density anomaly, but with two liquids with different local structures [67]. This observation allows us to conclude that the possibility of a liquid-liquid critical point is not limited to fluids with density anomalies - a conclusion supported by recent experiments for phosphorus [68-70] and triphenyl phosphite [71, 72].

In the case of water, the hypothesized liquid-liquid phase transition is probably hindered by inevitable freezing [52]. Indeed, it appears that the hypothesized liquid-liquid phase transition is below, or at least close to, the glass transition temperature. A recent simulation 
analysis of the orientational dynamics of water at fixed density [47] has shown that the temperature of dynamical arrest of the system, defined by the mode coupling theory, is relatively close in temperature and density to recent estimate of the liquid-liquid critical point [73]. However, as we have shown here, the study of the Widom line at temperatures above the liquid-liquid critical point could represent a useful way to investigate the inaccessible part of the supercooled phase diagram of water.

\section{Acknowledgments}

We thank our collaborators, P Kumar, M I Marqués, K Stokely, and M Yamada, and the NSF Chemistry Programme CHE 0096892 and CHE0404673 for support. GF also thanks the Spanish Ministerio de Educación y Ciencia (Programa Ramón y Cajal and Grant No FIS200403454).

\section{References}

[1] Cwilong B M 1947 Proc. R. Soc. A 190137

[2] Kanno H, Speedy R J and Angell C A 1975 Science 189881

[3] Brügeller P and Mayer E 1980 Nature 288569

[4] Mishima O, Calvert L D and Whalley E 1985 Nature 31476

[5] Mishima O 1994 J. Chem. Phys. 1005910

[6] Mishima O 1996 Nature 384546

[7] Suzuki Y and Mishima O 2002 Nature 491599

[8] Mishima O and Stanley H E 1998 Nature 396329 Mishima O and Stanley H E 1998 Nature 392164

[9] Brovchenko I, Geiger A and Oleinikova A 2003 J. Chem. Phys. 1189473 Brovchenko I, Geiger A and Oleinikova A 2005 J. Chem. Phys. 123044515

[10] Loerting T, Salzmann C, Kohl I, Mayer E and Hallbrucker A 2001 Phys. Chem. Chem. Phys. 35355

[11] Finney J L, Bowron D T, Soper A K, Loerting T, Mayer E and Hallbrucker A 2002 Phys. Rev. Lett. 89205503

[12] Loerting T, Schustereder W, Winkel K, Salzmann C G, Kohl I and Mayer E 2006 Phys. Rev. Lett. 96025702

[13] Debenedetti P G 1996 Metastable Liquids: Concepts and Principles (Princeton, NJ: Princeton University Press)

[14] Alba-Simionesco C, Coasne B, Dosseh G, Dudziak G, Gubbins K E, Radhakrishnan R and Sliwinska-Bartkowiak M 2006 J. Phys.: Condens. Matter 18 R15

[15] Faraone A, Liu L, Mou C Y, Yen C W and Chen S H 2004 J. Chem. Phys. 12110843

[16] Liu L, Faraone A, Mou C Y, Yen C W and Chen S H 2004 J. Phys.: Condens. Matter 16 S5403

[17] Liu L, Chen S H, Faraone A, Yen C W and Mou C Y 2005 Phys. Rev. Lett. 95117802

[18] Mallamace F, Broccio M, Corsaro C, Faraone A, Wanderlingh U, Liu L, Mou C Y and Chen S H 2006 J. Chem. Phys. 124161102

[19] Swenson J, Jansson H and Bergman R 2006 Phys. Rev. Lett. 96247802

[20] Chen S-H, Liu L, Fratini E, Baglioni P, Faraone A and Mamontov E 2006 Proc. Natl Acad. Sci. USA 1039012

[21] Mamontov E 2005 J. Chem. Phys. 123171101

[22] Jansson H, Howells W S and Swenson J 2006 J. Phys. Chem. B 11013786

[23] Lubchenko V, Wolynes P G and Frauenfelder H 2005 J. Phys. Chem. B 1097488 Fenimore P W, Frauenfelder H, McMahon B H and Young R D 2005 Physica A 3511

[24] See, for example, Swenson J 2006 Phys. Rev. Lett. 97189801 Cerveny S, Colmenero J and Alegría A 2006 Phys. Rev. Lett. 97189802 Chen S-H, Liu L and Faraone A 2006 Phys. Rev. Lett. 97189803

[25] Oleinikova A, Smolin N and Brovchenko I 2006 J. Phys. Chem. B 11019619 Brovchenko I, Krukau A and Oleinikova A 2006 Phys. Rev. Lett. 97137801

[26] See, for example, Iordanov T D, Schenter G K and Garrett B C 2006 J. Phys. Chem. A 110762

[27] Debenedetti P G 2003 J. Phys.: Condens. Matter 15 R1669

[28] Franzese G and Stanley H E 2002 J. Phys.: Condens. Matter 142201

[29] Franzese G and Stanley H E 2002 Physica A 314508

[30] Speedy R J and Angell C A 1976 J. Chem. Phys. 65851

[31] Angell C A, Oguni M and Sichina W J 1982 J. Phys. Chem. 86998

[32] Rasmussen D H and MacKenzie A P 1973 J. Chem. Phys. 595003 
[33] Debenedetti P G and Stanley H E 2003 Phys. Today 56 (6) 40 Stanley H E, Teixeira J, Geiger A and Blumberg R L 1981 Physica A 106260

[34] Hare D E and Sorensen C M 1987 J. Chem. Phys. 874840

[35] Pauling L 1960 The Nature of the Chemical Bond, and the Structure of Molecules and Crystals 3rd edn (Ithaca, NY: Cornell University Press)

[36] Soper A K and Ricci M A 2000 Phys. Rev. Lett. 842881

[37] Svishchev I M and Kusalik P G 1993 J. Chem. Phys. 993049

[38] Bosio L, Chen S-H and Teixeira J 1983 Phys. Rev. A 271468

[39] Pople J A 1951 Proc. R. Soc. A 205163

[40] Poole P H, Sciortino F, Essmann U and Stanley H E 1992 Nature 360324 Poole P H, Sciortino F, Essmann U and Stanley H E 1993 Phys. Rev. E 483799 Poole P H, Essmann U, Sciortino F and Stanley H E 1993 Phys. Rev. E 484605

[41] Schwegler E, Galli G and Gygi F 2000 Phys. Rev. Lett. 842429

[42] Saitta A M and Datchi F 2003 Phys. Rev. E 67 020201(R)

[43] Li F, Cui Q, He Z, Cui T, Zhang J, Zhou Q, Zou G and Sasaki S 2005 J. Chem. Phys. 123174511

[44] Mathias G and Tavan P 2004 J. Chem. Phys. 1204393

[45] Sciortino F, Geiger A and Stanley H E 1990 Phys. Rev. Lett. 653452 Canpolat M, Starr F W, Sadr-Lahijany M R, Scala A, Mishima O, Havlin S and Stanley H E 1998 Chem. Phys. Lett. 2949

Sciortino F, Geiger A and Stanley H E 1991 Nature 354218

[46] Laage D and Hynes J T 2006 Science 311832

[47] Kumar P, Franzese G, Buldyrev S V and Stanley H E 2006 Phys. Rev. E 73041505

[48] See, for example, the table of structural data in Martin Chaplin water website http://www.lsbu.ac.uk/water/phase. html and references therein

[49] D'Arrigo G, Maisano G, Mallamace F, Migliardo P and Wanderlingh F 1981 J. Chem. Phys. 754264

[50] Angell C A and Rodgers V 1984 J. Chem. Phys. 806245

[51] Balladares A L and Barbosa M C 2004 J. Phys.: Condens. Matter 168811

[52] Franzese G, Marqués M I and Stanley H E 2003 Phys. Rev. E 67011103

[53] Sastry S, Debenedetti P G, Sciortino F and Stanley H E 1996 Phys. Rev. E 536144

[54] Stokely K, Franzese G and Stanley H E 2007 in preparation

[55] Angell C A, Borick S and Grabow M 1996 J. Non-Cryst. Solids 207463

[56] Poole P H, Hemmati M and Angell C A 1997 Phys. Rev. Lett. 792281

[57] Xu L, Kumar P, Buldyrev S V, Chen S-H, Poole P H, Sciortino F and Stanley H E 2005 Proc. Natl Acad. Sci. 10216558

[58] Kumar P, Yan Z, Xu L, Mazza M G, Buldyrev S V, Chen S-H, Sastry S and Stanley H E 2006 Phys. Rev. Lett. 97177802

[59] Stanley H E, Kumar P, Xu L, Yan Z, Mazza M G, Buldyrev S V and Chen S-H 2007 Relation between the Widom line and the dynamic crossover in bulk water and in protein hydration water Proc. 8th Int. Conf. on Quasi-Elastic Neutron Scattering ed P E Sokol, H Kaiser, D Baxter, R Pynn, D Bossev and M Leuschner (Warrendale, PA: Materials Research Society) p 3

[60] Sciortino F, Poole P H, Essmann U and Stanley H E 1997 Phys. Rev. E 55727

[61] Trinh E and Apfel R E 1980 J. Chem. Phys. 726731

[62] Kumar P, Franzese G and Stanley H E 2007 Effect on the hydrogen bond network of water on crossing the Widom line Preprint cond-mat/0702108

[63] Stanley H E 1979 J. Phys. A: Math. Gen. 12 L329

Stanley H E and Teixeira J 1980 J. Chem. Phys. 733404

Stanley H E, Teixeira J, Geiger A and Blumberg R L 1981 Physica A 106260

Geiger A and Stanley H E 1982 Phys. Rev. Lett. 491749

[64] Raiteri P, Laio A and Parrinello M 2004 Phys. Rev. Lett. 93087801

[65] Saika-Voivod I, Sciortino F and Poole P H 2001 Phys. Rev. E 63011202

[66] Sastry S and Angell C A 2003 Nat. Mater. 2739

[67] Franzese G, Malescio G, Skibinsky A, Bulderev S V and Stanley H E 2001 Nature 409692

[68] Katayama Y, Mizutani T, Utsumi W, Shimomura O, Ya-makata M and Funakoshi K 2000 Nature 403170

[69] Katayama Y, Inamura Y, Mizutani T, Yamakata M, Utsumi W and Shimomura O 2004 Science 306848

[70] Monaco G, Falconi S, Crichton W A and Mezouar M 2003 Phys. Rev. Lett. 90255701

[71] Tanaka H, Kurita R and Mataki H 2004 Phys. Rev. Lett. 92025701

[72] Kurita R and Tanaka H 2004 Science 306845

[73] Brovchenko I, Geiger A and Oleinikova A 2005 J. Chem. Phys. 123044515 